ARTÍ́CULO DE HISTORIA Y EDUCACIÓN

\title{
La prensa y los rayos $X$ en México a finales del siglo XIX
}

\section{The press and the X-rays $n$ Mexico at the end of the XIX century}

Fernando de Alba-Quintanilla1*, Carlos A. de Alba-Guevara² y Gustavo A. Casian-Castellanos ${ }^{3}$

${ }^{1}$ Centro de Radiodiagnóstico, San Luis Potosí; ${ }^{2}$ Departamento de Imagen, Hospital MEDISCIN, San Luis Potosí; ${ }^{3}$ Departamento de Rayos X, Hospital Juarez de México, Ciudad de México. México

\section{RESUMEN}

La primera información acerca de los rayos $X$ que hemos encontrado apareció el 24 de enero de 1896 en el diario de la Ciudad de México La Patria. A partir de esa fecha, la información que difundió la prensa de la Ciudad de México acerca de los rayos $X$ fue extensa, exacta y suficiente para que, contando con el equipo necesario, se llegaran a reproducir los experimentos realizados por Roentgen y, con ello, poder realizar la fluoroscopia y obtener una radiografía. Fue así que, el 8 de marzo de 1896 el diario El Universal menciona que el Dr. Roberto Jofre ya estaba realizando estudios radiográficos experimentales en su Gabinete de Electricidad Médica. De estos primeros experimentos no tenemos ninguna evidencia. Las primeras radiografías conocidas fueron realizadas por el Dr. Roberto Jofre y mostradas en una conferencia el 3 de agosto de 1896. Queda abierta la posibilidad de que el Profesor Luis G. León también hubiera realizado tempranamente experimentos con los rayos $\mathrm{X}$.

Palabras clave: Radiología. Historia de la Medicina.

\section{ABSTRACT}

The first information regarding of the $X$ rays we have documentation appeared in the newspaper of Mexico City La Patria on January 24 of 1896. After that the information spread by the press of Mexico City was extense, trustworthy and enough to (with the right equipment) reproduce the experiments realized by Roentgen, perform fluoroscopy and 
also obtain a radiography. This was how in March 8 of 1896, the newspaper El Universal published that Dr. Roberto Jofre was already performing radiographic experiments in his medical electricity facility. We don't have evidence of the first's experiments. The first $x$ rays known performed by Dr. Roberto Jofre were shown in a lecture on August 3 of 1896. The possibility that the professor Luis G. Leon early performed experiments with the $\mathrm{X}$ rays is open but we don't have the evidence.

Key words: Radiology. History of Medicine.

\section{INTRODUCCIÓN}

A finales del siglo XIX, la ciencia pasó de ser exclusiva de los círculos eruditos a convertirse en un tema de interés común, dejó de ser analizada en círculos cerrados y empezó a difundirse a nivel popular, a ser entendida o, al menos, ser un motivo diario de conversación entre la población lectora y con frecuencia toda una trama de especulación y fantasía. Los términos técnicos y científicos se incorporaron a la lengua cotidiana. En forma paulatina, el lenguaje se adaptó, su contenido se simplificó y muy pronto los temas científicos empezaron a ser tratados fuera de las catedrales del conocimiento y por la gente letrada, el texto empezó a ser acompañado de ilustraciones. ${ }^{1}$.

El ascenso de la divulgación de la ciencia está ligado al aumento del nivel educativo de la población, y los medios de comunicación impresos, entre ellos la prensa, fueron el instrumento para la divulgación del conocimiento. La ciencia en su forma popularizada empezó a ocuparse de temas que despertaron el interés en el público lector. El periódico se convirtió en el principal medio masivo de comunicación, y adquirió un importante papel protagónico. Todo esto se vio beneficiado porque, en el panorama informativo, las innovaciones técnicas permitieron la impresión rápida y masiva.

La pasión por la información fue una de las características del siglo XIX. En la búsqueda y redacción de los artículos, el resultado final era que notificaban todo tipo de novedades, ciertas e inciertas. Los escritores fueron adaptándose a las exigencias de los lectores y viceversa. Las agencias noticiosas participaron en la promoción del carácter extranacional de la ciencia ${ }^{2}$.

Estas circunstancias favorecieron la rápida difusión del descubrimiento de los rayos $X$ por Roentgen en prácticamente todo el mundo. La difusión del descubrimiento fue muy rápida y sin barreras, tanto fue así que ese año de 1896 se publicaron más de 1,000 trabajos científicos y 48 libros sobre los rayos $X^{3}$. Roentgen descubrió los rayos $X$ en noviembre de 1895 cuando se encontraba estudiando los misteriosos rayos catódicos. Para ello utilizó sencillos aparatos que se podían encontrar en cualquier laboratorio de física o de electricidad, por modesto que fuera, o que se podían adquirir fácilmente en el comercio por ser instrumentos de uso habitual. Utilizando un tubo de Crookes, que tenía como fuente de energía eléctrica una bobina de Rumhkorff, y empleando una pantalla 
fluorescente elaborada artesanalmente con una hoja de cartón recubierta por cristales de patino cianuro de bario, que se utilizaba para identificar los rayos catódicos fuera del tubo, fue como descubrió los rayos X. Para evidenciarlos utilizó papel fotográfico común y corriente, y obtuvo fotografías de variados objetos. Un descubrimiento excepcional logrado con mucho ingenio y un mínimo de equipo $^{4}$.

\section{LA PRENSA EN MÉXICO A FINALES DEL SIGLO XIX}

En México, a finales del siglo XIX, la censura y persecución impuesta por el presidente Porfirio Díaz a los periodistas críticos al régimen les obligó a enfocar la información de los diarios hacia temas de alabanza al régimen o a contenidos no políticos. Díaz controlaba la prensa a través de dos medios: el patrocinio directo o mediante la aplicación de la Ley de Imprenta, esto es, la persecución ${ }^{5}$.

Una de las consecuencias de esto fue la difusión en la prensa de temas científicos y de las actividades académicas que se verificaban en la Ciudad de México. Se había establecido una especie de alianza entre las sociedades científicas y la prensa encargada de difundir los eventos de esas agrupaciones.

Los directivos de la prensa tuvieron que buscar, y encontrar, el camino para divulgar aquellos temas que despertaban más interés y que aumentaban el tiraje. La integración del sistema telegráfico y telefónico enriqueció a la prensa. Simultáneamente empezaron a aparecer las agencias noticiosas que difundían y permitían que la información proveniente de Europa y de otras partes del mundo se proporcionara en forma vertiginosa $^{3}$.

El 7 de marzo de 1896, el periódico El Tiempo publicó las cifras del censo de la República realizado en $1895^{6}$. La población total era de unos 12.400.000 habitantes y, de ellos, el 18\% eran alfabetos.

En la República Mexicana, el mayor número de diarios se publicaban en la Ciudad de México, en donde había un sinnúmero de periódicos y publicaciones circulando. Hacía 1892, en la Ciudad de México, se publicaban unos 20 diarios, aunque con seguridad circulaban varios más de manera informal. Los diarios tenían tirajes que variaban entre 800 y 20,000 ejemplares. Estos diarios tenían por lo general entre cuatro y seis páginas, aunque con relativa frecuencia las aumentaban a seis $\mathrm{u}$ ocho, por lo general los domingos. Los tirajes elevados de los diarios abarataron los costos, así, a afínales del siglo XIX, el precio de un diario en la Ciudad de México variaba entre 1 y $5 \mathrm{Cvs}^{2}$.

Las ilustraciones eras litográficas. La mayoría de los periódicos constaban de cuatro páginas, y la última de ellas se utilizaba con fines publicitarios, que se convirtió en la representación del espacio público.

En todos esos diarios, en alguna de las páginas intermedias había una sección de noticias extranjeras. Hacia 1896, este servicio era proporcionado a todos los periódicos por igual por la Agencia Cablegráfica y Telegráfica Mexicana de la que Albert Samson era el director. Había otras agencias que brindaban este servicio, entre ellas estaba la Agencia 
Agusti y la Agencia Godoy y Pratt ${ }^{7}$, pero eran menos relevantes.

La importancia de esto reside en que, siendo una sola la agencia de noticias que había en México, prácticamente los mismos boletines informativos que se recibían de Europa u otras partes del mundo eran enviados a todos los periódicos. Sin embargo, los diarios no tenían la obligación de publicarlos el día que los recibían, esto se regía de acuerdo al criterio de la redacción, que tenían la libertad de insertarlos en la fecha en que, de acuerdo a sus normas o intereses, lo consideraran conveniente ${ }^{7}$. Así es que, una nota proveniente del extranjero podía publicarse en un diario el día en que era recibida, y esa misma nota, con las mismas palabras, podía aparecer en otro diario en una fecha diferente, por decir algo, diez días después. En los diarios de México, los boletines en los que se hace referencia a Roentgen y su descubrimiento de los rayos $X$ se encuentra dispersa entre el 24 de enero y los últimos días del mes de marzo de 1896. Durante ese periodo, en ocho de los diarios a los que hemos tenido acceso (El Correo Español, El Monitor Republicado, El Nacional, La Patria, El Universal, La República, El Siglo Diez y Nueva y El Tiempo), se publicaron 44 artículos en los que se hace referencia a Roentgen o a los rayos X. A partir del mes de abril, las noticias relacionadas con los rayos $\mathrm{X}$ empezaron a disminuir, hasta que finalmente, en forma insensible, dejaron de aparecer.

\section{LAS PRIMERAS NOTICIAS ACERCA DE LOS RAYOS $X$}

La primera nota que hace referencia a los rayos $X$ y que hemos encontrado apareció en el periódico La Patria, uno de los diarios menores de la Ciudad de México, el viernes 24 de enero de 1896 (Fig. 1). En un segmento titulado «Noticias», que se localiza en la segunda columna de la página 3 , apareció la siguiente nota ${ }^{8}$.

Llama la atención lo temprano de la aparición de la noticia del descubrimiento de los rayos $\mathrm{X}$ en la prensa mexicana. Sin lugar a dudas, esta es la primera comunicación publicada al respecto, ya que, apenas un día antes, el 23 de enero, Roentgen (escrito como Routgen en esta y otras comunicaciones) había presentado su hallazgo en la Sociedad Físico Médica de Würzburg. Otro aspecto llamativo de este reporte inicial es que la noticia fue remitida de Londres, no de Alemania. Algunos otros boletines que encontramos también procedían de Inglaterra.

El siguiente reporte lo localizamos en El Monitor Republicano, el día 29 de enero9. En la sección relacionada con noticias varias del extranjero apareció la siguiente nota:

\section{El monitor republicano}

Miércoles 29 de enero. 1896. Página 2.

INVENTOS VALIOSOS.

El electricista Nicolás Tesla, que compite con Edison en talento inventivo acaba de idear una máquina con el nombre de oscilador...

...También ha producido una luz astral sutilísima $y$ de gran poder lumínico, cuyos rayos, en condiciones peculiares, tienen gran facilidad para penetrar ciertas substancias como la carne, y se ven detenidos por otras como los huesos, propiedades que la hacen preciosa para estudios de medicina $y$ 


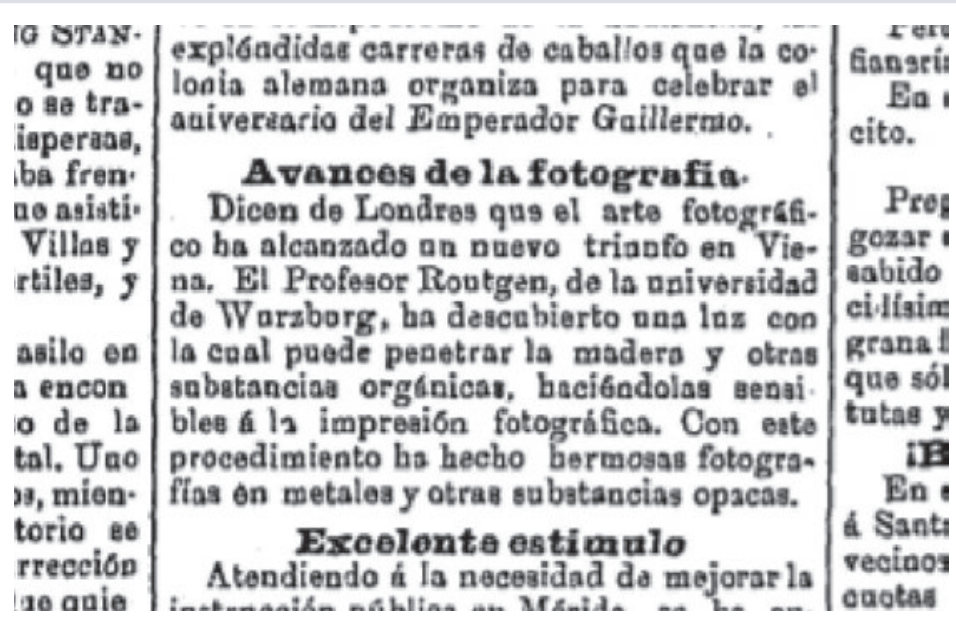

Figura 1. Primera notificación aparecida en la prensa de México en la que se informa acerca del descubrimiento de los ratos $X$ y que apareció el día 24 de enero de 1896 en el periódico La Patria.

cirujía [sic], pudiéndose localizar con gran precisión por el contraste de luz y sombra el punto donde se encuentra una bala que extraer o tumor interno que operar.

Las propiedades de esta luz se han aplicado en Alemania por el Profesor Routgen de la Universidad de Wursburg, con tan buen éxito, que pudo sacar el retrato de una joven, apareciendo a su lado el esqueleto de un individuo que la acompañaba.

Al final de esta nota se hace mención del «retrato de una joven, apareciendo a su lado el esqueleto de un individuo que la acompañaba», que más tarde fue publicado en primera plana en el diario El Universal, el 14 de febrero. Corresponde a la litografía de una pareja, una mujer en un sillón y, a su lado, un esqueleto contorneando tenuemente el cuerpo de un individuo ${ }^{10}$.

A partir del 1 de febrero se empezó a vislumbrar con certeza la aplicación de los rayos $\mathrm{X}$ a la medicina y cirugía, y a difundirlo. En El
Universal apareció un reporte titulado «Algo nuevo en la cirugía militar» ${ }^{11}$.

Así, durante la primera mitad del mes de febrero continuaron apareciendo en forma dispersa, en los diarios de la Ciudad de México, cablegramas con boletines relacionados con los rayos $\mathrm{X}$. Como hemos dicho, la gran mayoría de estos boletines se publicaban, de manera repetida, en diferentes diarios, aunque, con frecuencia, en diferentes fechas ${ }^{8-13}$. Es importante enfatizar que en la difusión que se hacía del nuevo descubrimiento con frecuencia se hablaba e insistía en que se trataba de «una nueva fotografía», al grado que en Berlín se formó una Asociación Central Fotográfica de Roentgen ${ }^{14}$.

En el mismo ejemplar de El Universal del 14 de febrero, en la página 3 se publica un extenso artículo acerca de los rayos $\mathrm{X}$ titulado «La doble vista comprobada» ${ }^{15}$. La información recabada y asentada en el presente artículo proviene de París (Fig. 2). 


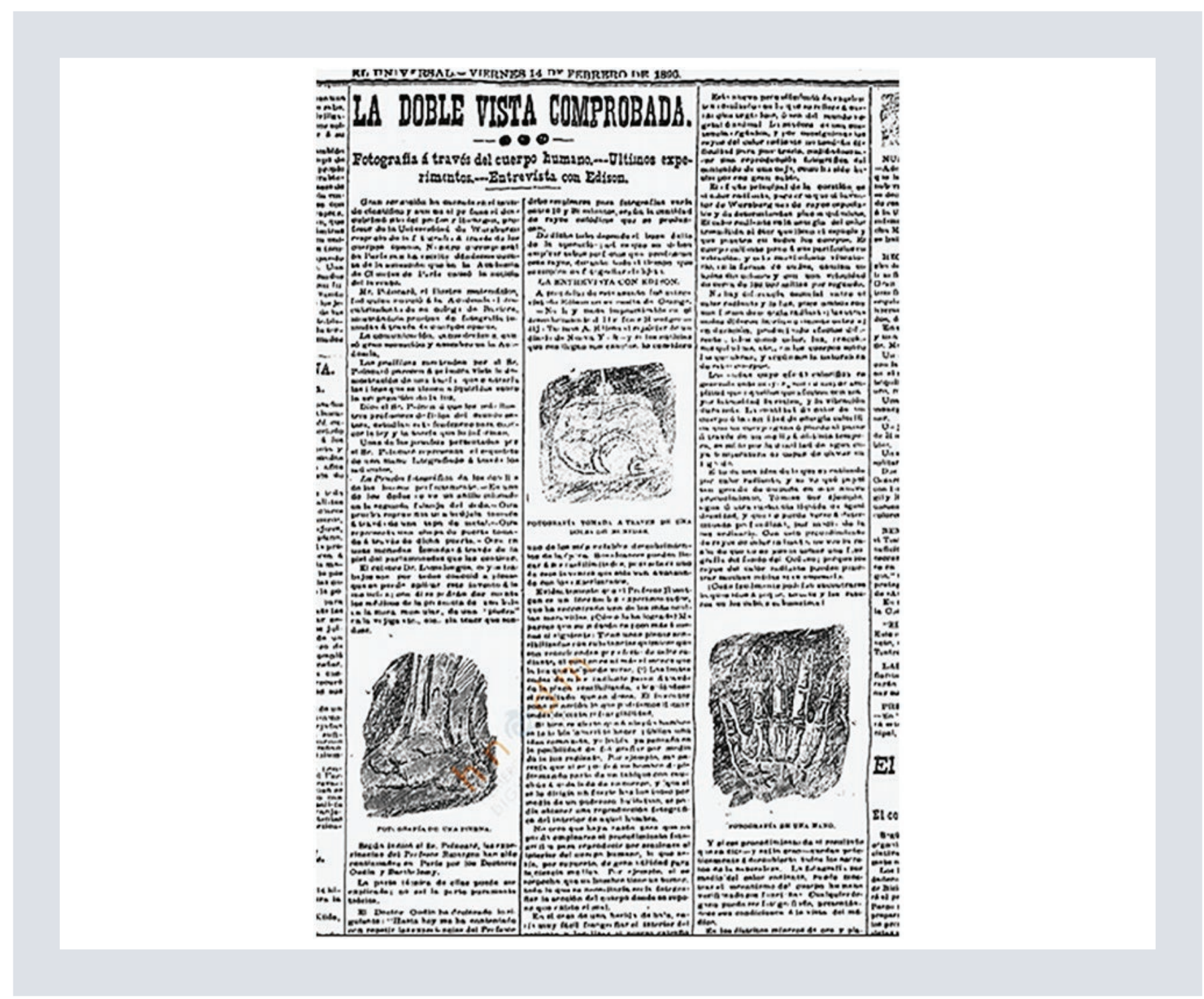

Figura 2. Extenso artículo aparecido en El Universal en el que, además de mostrar litografías representativas de unas radiografías, describe la manera en que se pueden producir los rayos $X$.

Este es un extenso artículo en donde se muestra la litografía de dos radiografías, una del tobillo y otra de la muñeca, así como la de una bolsa con monedas. A continuación, unos fragmentos del artículo:

...La parte técnica de ellas puede ser explicada, no así la parte teórica...

...La parte técnica es sumamente sencilla. Consiste en tomar una placa fotográfica ordinaria, envolverla en varias hojas de papel negro y después fijar dicha placa a una distancia de 10 centímetros del tubo que produce los rayos catódicos; en seguida se interpone entre el foco de luz y la placa el objeto u órgano del cual se desea obtener la permeabilidad.

Después basta hacer pasar una corriente eléctrica de una bobina de Ruhmkorff. Se hace pues pasar dicha corriente por el tubo disponiéndolo de tal modo que los rayos catódicos vengan a caer sobre la placa y el objeto, lo más perpendicularmente posible. 
De este artículo se puede rescatar lo siguiente:

- Primero, se describe en forma muy sencilla la manera en que se podía realizar una «fotografía de Roentgen», nada más fácil para quienes estaban familiarizados con el estudio de la electricidad y contaban con un tubo de rayos catódicos.

- El segundo aspecto es que hace mención de Edison y de las pantallas de fluoroscopia, en las que, para esa fecha, Edison era un experto. Había estudiado un gran número de compuestos químicos y fabricado sus propios fluoroscopios (Vitascopio).

- El tercero de ellos es que se podían producir rayos $\mathrm{X}$ y obtener radiografias, aun desconociendo la manera en que actuaban estos. En ese momento, las hipótesis acerca de su origen estaban lejanas a la realidad, esto propiciaba que la fantasía y el pensamiento mágico se mezclara con la realidad.

A lo largo del mes de febrero fue apareciendo nueva información, como el reto que lanzó W.R. Hearst a Edison para que obtuviera una radiografía del cerebro. El experimento del Dr. E. von Neusser, quien fue el primero en tomar radiografías de cálculos vesiculares, demostró que los cálculos de colestrina (colesterol) eran radiolúcidos, en contraste con aquellos que contienen calcio y que son radiopacos $^{16-25}$.

Los lectores de los diarios eran bombardeados por notas breves, o extensas, que ensalzaban las maravillas de la misteriosa fotografía de Roentgen. El 25 de febrero, en El Universal, se mostró una litografía de Roentgen ${ }^{26}$, que corresponde al primer retrato conocido de este, en México.
Algunos de estos artículos podrían ser motivo de un análisis más exhaustivo del que brinda este espacio, muchos eran informativos y, con frecuencia, describían el equipo y el procedimiento para lograr una radiografía.

\section{FATANSÍA DE LOS RAYOS X EN LA PRENSA MEXICANA}

El Universal es el diario en el que encontramos el mayor número de artículos acerca de los rayos $\mathrm{X}$ en aquel primer año, sin embargo, como hemos dicho, también aparecían noticias en otros diarios con cierta regularidad. Algunos de los artículos eran informativos, pero la ignorancia dejaba abierta la puerta a la imaginación y mucho de lo informado caía en el terreno de la fantasía, era especulativo, sin fundamento y lleno de falsedades. Lo atractivo para los diarios era que despertaba mucho el interés popular.

En el repaso de la literatura hemos de mencionar unos pocos reportes, como el que apareció el 16 de febrero en El Monitor Republicano ${ }^{27}$. Se trata de una divagación sobre la facilidad para traspasar paredes y puertas, con la finalidad de sorprender a los maridos infieles con una simple cámara fotográfica de rayos X. Sin lugar a dudas, un gran número de los lectores creían a pie puntillas lo publicado.

Otro de los artículos francamente fantasioso apareció en el El Universal a finales de febrero y se refiere al Dr. Carleton Simon, de Nueva York, quien exhibía una litografia de su cerebro $^{28}$. El Dr. Simon se convirtió en un psiquiatra y criminalista afamado.

O bien, el artículo de La Luz Negra, de Gustave Le Bon, un investigador serio $\mathrm{y}$ 
reconocido, quien estaba realizando «experimentos de fotografía a través de los cuerpos opacos, pero sin ayuda de la luz de origen cathódico». Refería que una «luz negra» emergía de la flama de una lámpara de aceite, de la luz natural o de un tubo de rayos catódicos, y que esta energía pasa a través de los cuerpos opacos, por ejemplo, una placa de acero, y podría sensibilizar a una placa fotográfica colocada en el interior ${ }^{16,29}$. Años después reclamaba prioridad en el descubrimiento de los rayos $\mathrm{N}$ por Blondlot. Al final se demostró que ni la luz negra ni los rayos $\mathrm{N}$ existían $^{30}$.

Uno más, el barón de Reichenbach, como muchos otros espiritistas, encontró en los rayos $\mathrm{X}$ una posible explicación racional al fenómeno de la clarividencia pudiendo registrar en placas fotográficas los pensamientos, sentimientos o sueños, lo que apareció publicado en un artículo titulado «Los magos y la fotografía invisible» ${ }^{31}$. No importó que el noble barón hubiera fallecido en 1869 , se le atribuía eso...y más.

\section{LOS PRIMEROS INVESTIGADORES MEXICANOS DE LOS RAYOS $X$. UN PRELUDIO}

La prensa daba parte de que, el domingo primero de marzo de 1896, en la Sociedad Científica Antonio Álzate, el Prof. Luis G. León impartía la primera conferencia en México acerca de los rayos $X$, con el título «La fotografía a través de los cuerpos opacos» ${ }^{32-34}$. El reportaje de la sesión dice:

...siendo de actualidad el asunto a través de los cuerpos opacos, suplicaba que se le permitiera presentar un pequeño estudio acerca de esa cuestión reservando para otra sesión el trabajo de química que tenía prometido.

El Sr. León Hizo público su agradecimiento a la señorita. Rafaela Suarez, Directora de la Escuela Nacional, que le ha proporcionado toda clase de elementos para la continuación de sus experimentos.

La conferencia apareció mencionada en el diario El Nacional el día 4 de marzo y en $E l$ Universal al día siguiente.

También el 5 de marzo, en el diario El Nacional, al final de un extenso artículo titulado «La luz Negra. Los rayos de Roentgen». Hay que resaltar una frase que se encuentra perdida entre tantas líneas, al final del artículo dice $^{16}$ :

... En México se han hecho ya algunas experiencias de tan interesante descubrimiento, pero de esto hablaremos en nuestro próximo artículo.

A la fecha no hemos podido localizar el artículo aludido.

\section{NOTICIA SORPRENDENTE}

El siguiente domingo, el 8 de marzo, apareció en El Universal un breve artículo con información a toda luz significativa, relevante y de gran trascendencia para la radiología mexicana. En este artículo se describe que para esa fecha, en México, el Dr. Roberto Jofre ya había realizado experimentos con rayos $\mathrm{X}$ y obtenido algunas radiografías (Fig. 3).

La siguiente es la nota aparecida en el diario ${ }^{35}$ : 


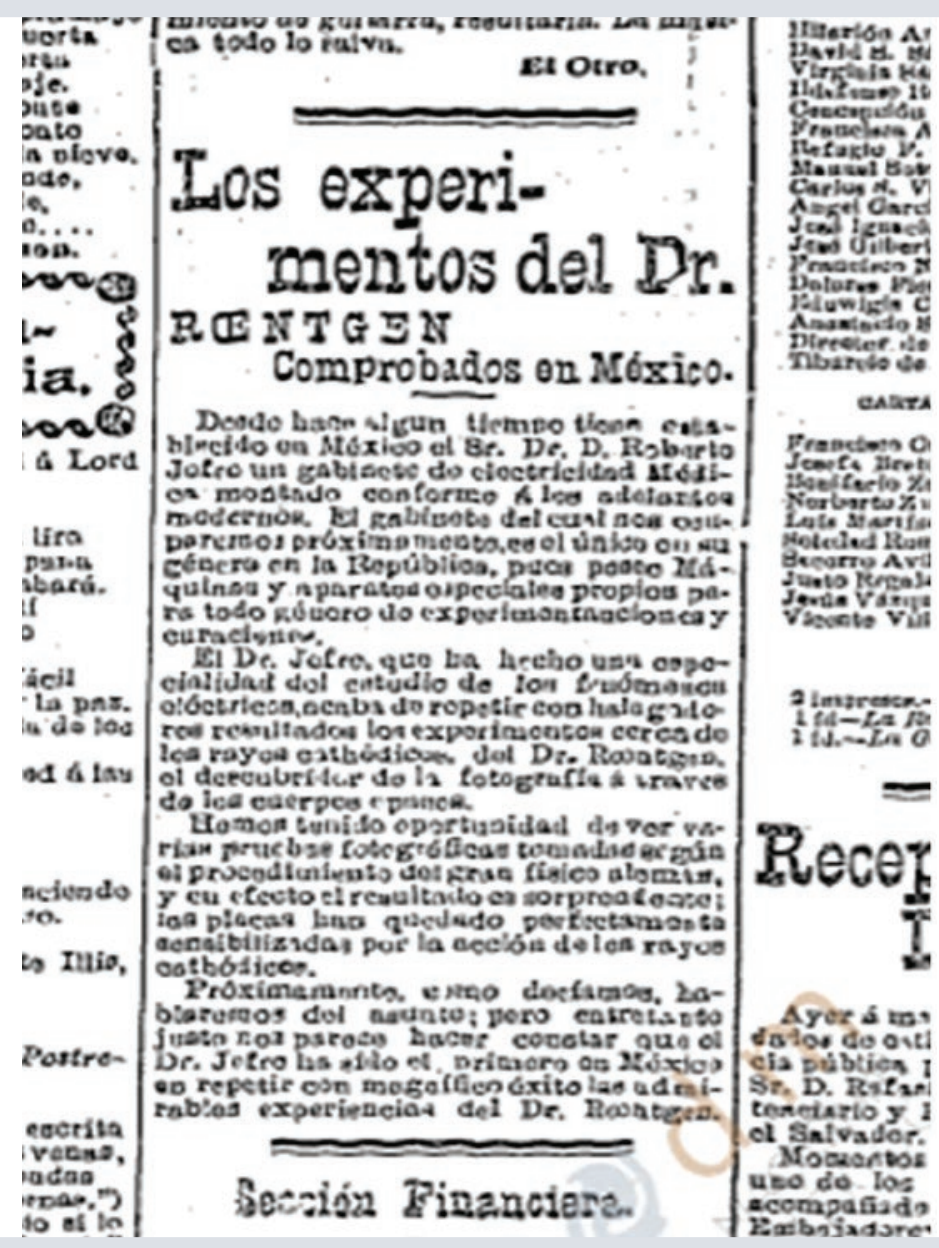

Figura 3. Nota aparecida en El Universal el 8 de marzo de 1896, en la que se informa que, en la Ciudad de México, el Dr. Roberto Jofre estaba realizando estudios radiológicos.

EL UNIVERSAL. Domingo 8 de Marzo de 1896, Página 2.

\section{LOS EXPERIMENTOS DEL DR. ROENT- GEN COMPROBADOS EN MÉXICO.}

Desde hace algún tiempo tiene establecido en México el Sr. Dr. Roberto Jofre un Gabinete de Electricidad Médica montado conforme a los adelantos modernos. El gabinete del cual nos ocuparemos próximamente es el único en su género en la República, pues posee máquinas y aparatos especiales propios para todo género de experimentaciones y curaciones.
El Dr. Jofre, que ha hecho una especialidad del estudio de los fenómenos eléctricos, acaba de repetir con halagadores resultados los experimentos cerca (sic) de los rayos catódicos del Dr. Roentgen, el descubridor de la fotografía a través de los cuerpos opacos.

Hemos tenido la oportunidad de ver varias pruebas fotográficas según el procedimiento del gran físico alemán, y en efecto el resultado es sorprendente; las placas han quedado perfectamente sensibilizadas por la acción de los rayos cathodicos. 
Próximamente, como decíamos, hablaremos del asunto; pero entre tanto justo nos parece hacer constar que el Dr. Jofre ha sido el primero en México en repetir con magnifico éxito las admirables experiencias del Dr. Roentgen.

La comunicación anterior nos parece suficiente evidencia acerca de que, desde temprana época, en los primeros días de marzo, el Dr. Roberto Jofre ya estaba realizando estudios radiológicos experimentales en la Ciudad de México, lamentablemente no se conoce ninguna radiografía fechada de este trabajo.

\section{EL GABINETE DE ELECTRICIDAD MÉDICA DEL DR. ROBERTO JOFRE EN LA PRENSA}

Acerca del trabajo a nivel privado del Dr. Roberto Jofre se ha mencionado con anterioridad que en 1890 organizó un gabinete de electricidad médica ${ }^{36}$, pero, atendiendo a los anuncios publicitarios aparecidos en la prensa, este Gabinete de Electricidad Médica ya estaba funcionando desde 1887 y era atendido por el Dr. José Manuel Jofre (ignoramos el parentesco). Podemos suponer que el Dr. Roberto M. Jofre, una vez titulado como Médico en 1890 y junto con el Dr. José Manuel Jofre, reorganizó un Gabinete de Electricidad Médica que ya funcionaba desde 1887 y se encontraba establecido en la calle de Rebeldes 5, casi esquina con San Juan de Letrán (hoy Artículo 123, casi esquina con Eje Central Lázaro Cárdenas) 37,38. Más tarde, el gabinete se ubicó en el número 15 de la misma calle de Rebeldes ${ }^{39}$.

Esta información se sustenta en algunos de los múltiples anuncios publicitarios aparecidos en la prensa a lo largo del tiempo (Fig. 4).
El primer anuncio publicitario del Gabinete de Electricidad Médica de los Dres. Jofre que hemos encontrado apareció el 22 de marzo de 1891, y en el encabezado y primera líneas refiere:

\section{GABINETE DE ELECTRICIDAD MÉDICA}

DE LOS DRES. JOFRE, REBLDES NUMERO 5.

Los anuncios publicitarios del gabinete de los Dres. Jofre estuvieron apareciendo en forma intermitente en la prensa, en diferentes diarios, a lo largo de los años. En uno de ellos, aparecido en 1894, se menciona que el Gabinete de Electricidad Médica había sido establecido, como ya se dijo, desde $1887^{40}$.

En una transcripción textual de este anuncio:

EL UNIVERSAL. Lunes 6 de agosto de 1894.

\section{GABINETE DE ELECTRICIDAD MÉDICA}

Establecido desde 1887 y recomendado en razón de su magnífico arsenal por los más distinguidos médicos de México y de los Estados.

Este establecimiento se encarga de componer aparatos eléctricos para usos médicos, de hacerlos iguales a los extranjeros y de agenciar su pedido a Europa o a los Estados Unidos.- Dr. José Manuel Jofre.- Dr. Roberto Jofre.

Servicio gratuito a los pobres.

A partir de junio de 1895, en los anuncios publicitarios del Gabinete de Electricidad 


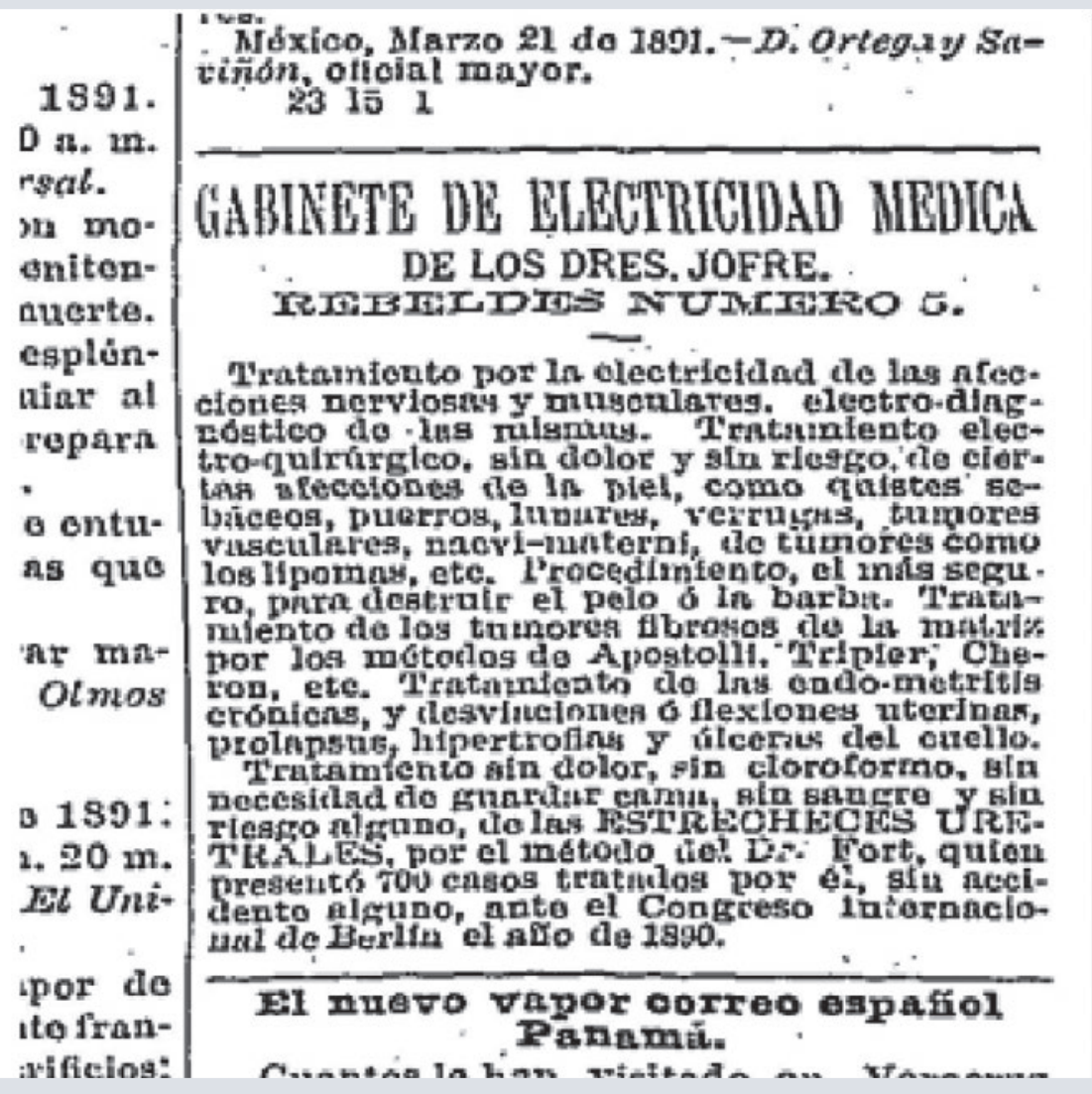

Figura 4. Publicidad aparecida en el diario El Universal el 21 de marzo de 1891. En la publicidad se anuncia el Gabinete de Electricidad Médica de los Dres. Jofre, ubicado en Rebeldes número 5, una calle del centro de la ciudad.

Médica, ya no se hace mención de «los Dres. Jofre», únicamente aparece el nombre del Dr. Roberto Jofre, sin haber encontrado mayor información al respecto.

El 19 de marzo de ese mismo año de 1896 apareció un artículo que ocupada la casi totalidad de la página de El Universal (Fig. 5), dedicado al Gabinete de Electricidad Médica del Dr. Roberto Jofre ${ }^{41}$.

A continuación se extractan algunos fragmentos del artículo:

EL UNIVERSAL. Jueves 19 de Marzo de 1896.

\section{EL GABINETE DE ELECTRICIDAD MÉDICA DEL DR. ROBERTO JOFRE.}

...Una de las aplicaciones de la electricidad y seguramente la más útil de todas, supuesto que se refiere directamente a la propia salud y conservación, es la Electroterapia. Pero entre la electroterapia actual y la electroterapia antigua (la electroterapia digamos de hace 15 años) media la misma distancia que entre la vela de sebo y el foco eléctrico no es exagerada, sino muy deficiente, por el contario.

...Este profesor (refiriéndose al Dr. Roberto Jofre), a fuerza de inquebrantable perseverancia, de constante estudio y de arduo trabajo, ha logrado dotar a la capital de un gabinete electroterápico, en 
donde se encuentran todos los vastísimos recursos que la ciencia eléctrica puede suministrar a la terapéutica.

El gabinete está dividido en tres departamentos. En el primero, que es representado por la lámina 1, están los generadores de energía mecánica y los dinamos y alternadores necesarios para transformarla en energía eléctrica.

... el doctor, venciendo todo género de dificultades, ha logrado implantar en una sección de su instituto todos los aparatos para producción de intensas corrientes o intensos campos electromagnéticos de altísima tensión y enorme frecuencia.

...Hablamos después de las experiencias de Roentgen. El Doctor me dijo que está trabajando con la colaboración de los Sres. Profesores Fernando Ferrari Pérez, Manuel R. Gutiérrez y de los Sres. Guillermo Knight, entendido electricista, Luis Labadie, hábil fotógrafo y los jóvenes Felipe y Julián Sierra, preparadores sustitutos de física y química respectivamente en la Escuela N. Preparatoria en la reproducción de dichas experiencias. Me dijo que estaba tropezando con dificultades casi insuperables por falta de buenos tubos de Lenard, por más que disponga de la magnífica colección de la Escuela Preparatoria, que en su afán por el progreso le haya facilitado su digno Director; pero que sin embargo ya había obtenido resultados alentadores y me mostró algunas placas perfectamente bien sensibilizadas por los rayos $X$ a través de láminas de madera de varios centímetros de grueso.

...La prosecución de estos experimentos de investigación y los que constituyen uno de los ideales que persigue el Doctor Jofre, en el perfeccionamiento de la anestesia por la electricidad, son dos grandes ilusiones que lo animan ahora que se trata de encargarlo del servicio de Electroterapia en el Hospital de San Andrés, sobre cuya interesante cuestión recaerá próximamente.
La extensión que tomado este artículo me impidió hablar de los demás departamentos del Gabinete del Dr. Jofre.

\section{Un electricista.}

Uno de los aspectos interesantes del artículo es que, aunque sea por medio de litografías, nos muestra varios aspectos del interior del gabinete. La información que aporta el artículo acerca del Gabinete del Dr. Roberto Jofre es extensa, casi toda ella relacionada con aspectos de equipamiento y operativos. Se ensalzan la importancia de la electricidad médica y su aplicación en beneficios de la salud, los conocimientos precisos de física que se debían de tener para lograr una efectiva terapia que en ese momento tenía más importancia que los recién descubiertos rayos, y con justa razón. Por supuesto que hace mención de los rayos $X$, y deja en claro que en México ya se habían iniciado los experimentos con estos. Resalta la fragilidad de los tubos catódicos y nos aclara que algunos de estos tubos le eran facilitados por los laboratorios de la Escuela Nacional Preparatorio. Finalmente menciona que había el proyecto de la instalación de un gabinete de electricidad médica en el Hospital General de San Andrés del que el Dr. Jofre sería el encargado.

De las personas señaladas como colaboradores del Dr. Jofre, ninguna se dedicó, ni figuró, en el ejercicio de la actividad radiológica, excepto el Ing. Ferrari Pérez, quien es mencionado en un artículo sobre los rayos $X$ escrito por el Ing. Gilberto Crespo y Martínez y más tarde en la Tesis del Dr. Amador Zafra ${ }^{42,43}$.

A mediados de diciembre de 1896, el Dr. Jofre trasladó el Gabinete de Electricidad Médica 


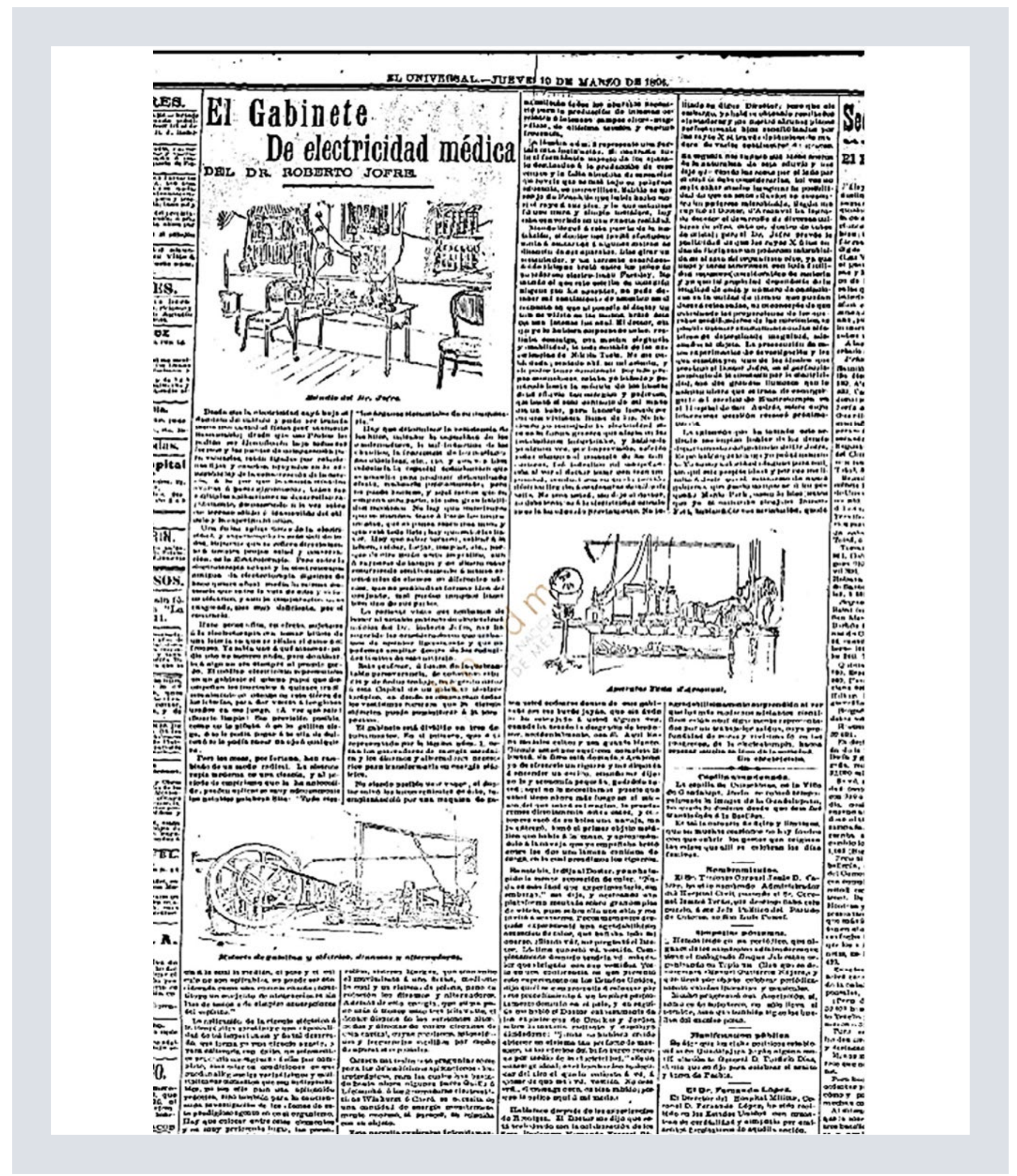

Figura 5. Página 4 de El Universal, del día 19 de marzo de 1896, en el que el que se describe el Gabinete de electricidad médica del Dr. Roberto Jofre. 
de la calle de Rebeldes a la calle de Xicotencatl $^{44-47}$ en la parte baja de una de las calles colindantes del Hospital de San Andrés, cuyo frente daba hacia la calle de Tacuba, en pleno centro de la Ciudad de México (más tarde en el sitio en donde estuvo el Hospital de San Andrés se construyó el Palacio de Comunicaciones y en la actualidad funciona el Museo Nacional del Arte) (Fig. 6).

\section{EPÍLOGO. UNA RUTA POR EXPLORAR}

Una líneas atrás escribimos que, el 5 de marzo de 1896, el Prof. Luis G. León presentó en la Sociedad Científica Antonio Álzate la conferencia «La fotografía a través de los cuerpos opacos ${ }^{32-34}$.

En 1896, Luis G. León era Profesor de Física de la Escuela Nacional Preparatoria y, por supuesto, miembro de la Sociedad Científica Antonio Álzate. La conferencia sustentada no aparece publicada en las Memorias y Revista de la Sociedad Científica Antonio Álzate Volumen 9 (1895-1896), ni en el 10 (1896-1897) ni en los tres volúmenes subsecuentes. El texto de la ponencia no aparece por ningún lado, no la hemos localizado en ninguno de los archivos consultados. Hay que señalar que no todas las conferencias que se presentaban eran aceptadas para ser publicadas en la revista.

El punto crítico reside en que el Prof. León, siendo maestro de Física, debía de conocer el asunto de la electricidad en detalle y suponemos que estaba al tanto del descubrimiento de los rayos X. Era aficionado a la fotografía y era maestro en la Escuela Nacional Preparatoria y, de acuerdo a lo referido en líneas anteriores, la Escuela Nacional Preparatoria facilitaba algunos de los tubos de Crookes al Dr. Jofre, para sus experimentos, de tal manera que no sería remoto que el Prof. León hubiera experimentado también con esos tubos y logrado alguna radiografía. Esta es una especulación, pero es una posibilidad que habrá de investigarse hasta agotar recursos y concluir con pruebas si acaso el Prof. Luis G. León llegó a obtener alguna radiografía experimental antes de las ahora conocidas, que fueron realizadas por el Dr. Roberto Jofre.

Aún más. El 8 de noviembre de 1896, en la sesión mensual oficial de la Sociedad Científica Antonio Álzate, al término de la presentación de las conferencias, hubo una demostración de fluoroscopia. Ni las memorias de la Sociedad, ni los artículos de la prensa alusivos al evento aportan mucha información al respecto. Dos periódicos nacionales $(E l$ Universal y El Nacional) relatan el evento ${ }^{48-50}$ :

...Se levantó la sesión, a la que concurrieron los socios González (Don Camilo A.), Aguilar, Oropeza, Rodríguez Rey, Cícero, Mendizábal (Don Joaquín), Galindo y Villa, León, Moreno, Vergara Lope, Mendizábal (Don José), Lozano y Castro, Cosío Villaseñor, Uribe, Troncoso y Torres Torija.

En seguida se pasó a sesión de experimentación con el Fluoroscopio de Edisson, quedando todos los socios sumamente complacidos de los preciosos resultados del descubrimiento de Roentgen.

$\mathrm{Ni}$ en los artículos ni en la memoria de la Sociedad se menciona el nombre de quien realizó los experimentos. Pero, como en la lista de los asistentes no se encuentra el Dr. Jofre, pero sí se menciona al Profesor Luis G. 


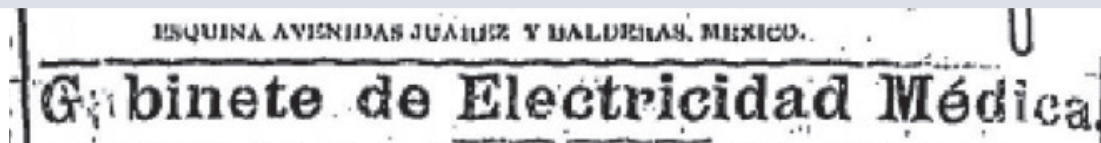

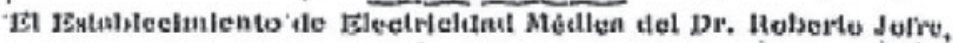

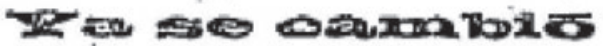

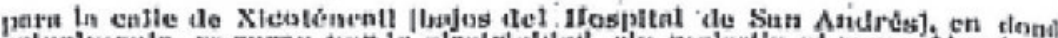

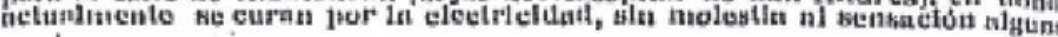 \\ DEBBLIDAD, NGURASTENIA \\ y sin hacer canin, sh operación cruents, sin dolor y sln rlestgo nilguno, \\ LAS FSTIRECEECIDS URETRAIES, \\ esofoginues, rectales, ute.

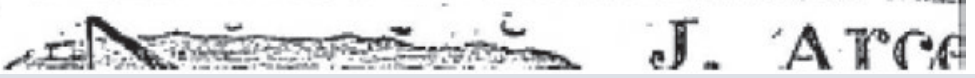

Figura 6. Anuncio en la prensa relacionado con el Gabinete de Electricidad Médica del Dr. Jofre encontrado en El Universal del día 15 de diciembre del 1896, en donde se informa que «El Establecimiento de Electricidad Médica del Dr. Roberto Jofre ya se cambió para la calle de Xicoténcatl [bajos del Hospital de San Andrés".

León, podemos postular la hipótesis que en esa época, este último, también realizaba experimentos de fluoroscopia.

El Profesor Luis G. León tenía como afición la fotografía. Fue autor de varios libros de astronomía y unos pocos y pequeños libros de fotografía entre los que se encuentran Cien experimentos de óptica, Algunas aplicaciones de la fotografía a la astronomía y uno más titulado $L a$ fotografía sin laboratorio.

\section{CONFLICTO DE INTERESES}

Los autores declaran que no existe conflicto de intereses.

\section{RESPONSABILIDADES ÉTICAS}

Protección de personas y animales. Los autores declaran que para esta investigación no se han realizado experimentos en seres humanos ni en animales.
Confidencialidad de los datos. Los autores declaran que en este artículo no aparecen datos de pacientes.

Derecho a la privacidad y consentimiento informado. Los autores declaran que en este artículo no aparecen datos de pacientes.

\section{BIBLIOGRAFÍA}

1. Panza M, Presas A. La divulgación del ciencia en el siglo XIX: La obra de Flammarion. Quark 2002. Disponible en: Quark.prbb.org.

2. Ruiz Castañeda MC, Lombardo García I, Camarillo Carbajal MT. La Prensa pasado y presente de México. Universidad Nacional Autónoma de México. México; 1990.

3. Phillips CES. Bibliography of X-Ray literatura and research, 1896-1897: Being a ready reference index to the literatura on the subject of Roentgen or X-Rays. «The Electrician» Printing And Publishing Co. London, England.

4. Klickstein HS, Wilhelm Conrad Roentgen. On a new kind of rays. Mallinckrodt Classics of Radiology. 1966.

5. Valadés J.C. El Porfirismo. Historia de un régimen. Fondo de Cultura Económica. México; 2015.

6. El censo de la República. El Tiempo. N. ${ }^{\circ} 3747,7$ de marzo de 1896. Página 1.

7. Por el Cable. Agencia Agusti. Telegramas para El Nacional. Agencia Godoy y Prat. Por la Línea del Ferrocarril Central. El Nacional. 8 de marzo de 1886. Página 3.

8. Avances de la fotografía. La Patria. N. ${ }^{\circ} 5770,24$ de enero de 1896. Página 3.

9. Inventos valiosos. El Monitor Republicano. N.o 25, 29 de enero de 1896. Página 2.

10. La fotografía a través de los cuerpos opacos. El Universal. N. ${ }^{\circ} 33,14$ de febrero de 1896. Página 1.

11. Algo nuevo en la cirugía militar. El Universal. N. ${ }^{\circ} 23,1$ de febrero de 1896 Página 3. 
12. Excelentes resultados. Los experimentos del descubrimiento del Dr. Roentgen. El Monitor Republicano. Año XLVI, N. 28,1 de febrero de 1896. Página 3, 4.

13. Dos experimentos del descubrimiento del Doctor Roentgen. El Siglo Diez y Nueve. N. ${ }^{\circ} 17,431,1$ de febrero de 1896. Página 3.

14. La Asociación Central Fotográfica de Roentgen. El Monitor Republicano. Año XLVI, N. ${ }^{\circ} 37,12$ de febrero de 1896. Página 4.

15. La doble vista comprobada. Fotografía a través del cuerpo humano. Últimos experimentos. Entrevista con Edison. El Universal. N. ${ }^{\circ} 33,14$ de febrero de 1896. Páginas 3.

16. La Luz Negra. Los Rayos de Roentgen. El Nacional. N. ${ }^{\circ} 207,5$ de marzo de 1896.

17. Luz a través de los cuerpos. El Monitor Republicano. Año XLVI, N. ${ }^{\circ} 40$, 15 de febrero de 1896. Página 2.

18. Fotografía del cerebro. El Universal. N. ${ }^{\circ} 39,21$ de febrero de 1896. Página 3.

19. Las maravillas del descubrimiento Roentgen. El Universal. N. ${ }^{\circ} 40,22$ de febrero de 1896. Página 5.

20. Alrededor del Mundo. Como se hace la radiografía a través de los cuerpos opacos. El Monitor Republicano. N. ${ }^{5}$ 56, 6 de marzo de 1896. Página 1.

21. Los rayos cathódicos (sic). El descubrimiento del Dr. Roentgen. Sección Extranjera. Los magos y la fotografía de lo invisible. El Universal. N. ${ }^{\circ} 43$, 26 de febrero de 1896. Página 3.

22. Notas Científicas. La primera aplicación de los rayos Roentgen en la cirugía. El Universal. N. ${ }^{\circ}$ 60, 16 de marzo de 1896. Página 5.

23. Charla de los Domingos: Hablando de los grandes descubrimientos. El Monitor Republicano. N. ${ }^{\circ}$ 59, 8 de marzo de 1896. Página 1.

24. El petróleo y la nueva fotografía. El Monitor Republicano. N.$^{\circ}$ 64, 14 de marzo de 1896. Página 1.

25. El valor de los rayos Roentgen en cirugía. El Nacional. N. ${ }^{\circ} 111,11$ de marzo de 1896. Página 2.

26. El Dr. Roentgen (litografía). Sección Extranjera: El Dr. Roentgen. El Universal. N. ${ }^{\circ} 42,25$ de febrero de 1896. Páginas 1, 4 .

27. Charla de los Domingos. El Monitor Republicano. N. ${ }^{\circ} 41,16$ de febrero de 1896. Página 1.

28. Los rayos Roentgen. El Cerebro del Dr. Simón. El Universal. N. ${ }^{\circ} 42,25$ de febrero de 1896. Página 3.

29. Las sombras radiográficas. La Luz negra. A propósito del gran descubrimiento de Roentgen. El Universal. N. ${ }^{\circ} 45,28$ de febrero de 1896. Página 6

30. De Alba Q.F. Blondlot y los rayos N: Acerca de una nueva especie de luz. Rev Méx Radiol. 2001;55 (1):31-4.

31. Los magos y la fotografía de lo invisible. La fotografía a través de los cuerpos opacos y la telepatía. El Monitor Republicano. N. ${ }^{\circ} 50,27$ de febrero de 1896 . Página 2.
32. León Luis G. Trabajos. La fotografía a través de los cuerpos opacos. Revista Científica y Bibliográfica de la Sociedad Científica Antonio Álzate. 18961897;3-41:16

33. Sociedad Científica «Antonio Álzate». El Nacional. N. ${ }^{\circ} 206,4$ de marzo de 1896. Páginas 1-2.

34. En la Sociedad Álzate. El Universal. N. ${ }^{\circ}$ 50, 5 de marzo de 1896. Página1.

35. Los experimentos del Dr. Roentgen comprobados en México. El Universal. N. ${ }^{\circ} 53,8$ de marzo de 1896. Página 2.

36. Nuestra Portada. Dedicatoria. Dr. Roberto Jofre. Rev Méx Radiol. 1965;49:182.

37. El Gabinete de Electricidad Médica de los Dres. Jofre. El Universal. N. ${ }^{\circ} 66$, 22 de marzo de 1891. Página 4.

38. Gabinete de Electricidad Médica de los Dres. Jofre. El Universal. N. ${ }^{\circ}$ 2, 22 de abril de 1891. Página 4.

39. Calificaciones hechas por las Juntas de Profesiones y Patente durante los años Fiscales de 1894 a 1895 y de 1895 a 1896. Dirección de Contribuciones Directas del Distrito Federal. México. Tipografía de la Oficina Impresora de Estampillas. Palacio Nacional. 1894.

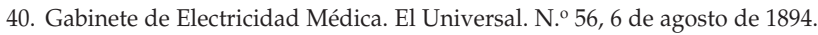
Página 4.

41. El Gabinete de Electricidad Médica del Dr. Roberto Jofre. El Universal. N. ${ }^{\circ}$ 62; 19 de marzo de 1896. Página 4.

42. De Alba Quintanilla F, Casian-Castellanos GA, De Alba-Guevara CA. Escribiendo la historia de la radiología en México. Las primeras radiografías. Rev Hosp Jua Mex. 2017;84(2):114-8.

43. De Alba GCA, De Alba QF, Casián CG. La Ciudad de México, cuna del primer estudio radiológicos en la República Mexicana. Gac Med Mex. 2018;154:1-5

44. Gabinete de Electricidad Médica. En Mudanza. El Universal. N. ${ }^{\circ} 205,4$ de diciembre de 1896. Página 5.

45. Gabinete de Electricidad Médica. En Mudanza. El Universal. N. ${ }^{\circ} 271,12$ de diciembre de 1896. Página 8.

46. Gabinete de Electricidad Médica. Ya se cambió. El Universal. N. ${ }^{\circ} 272,15$ de diciembre de 1896. Página 6.

47. Por medio de una radiografía. El Imparcial. N. ${ }^{\circ} 2295.1$ de Enero de 1903: Página 3.

48. León Luis G. Fluoroscopio. Revista Científica y Bibliográfica de la Sociedad Científica Antonio Álzate. 1896-1897;3-4:43-4.

49. Sociedad Científica "Antonio Álzate». Sesión del $1^{\circ}$ de marzo de 1896. El Nacional. N. ${ }^{\circ} 2062,4$ de marzo de 1896. Págs. 1-2.

50. En la Sociedad «Álzate». Sesión interesante. El Universal. N. ${ }^{\circ}$ 50, 5 de marzo de 1896. Pág. 1. 